\title{
Pituitary aspergillosis presenting as macroadenoma: case report and review of literature
}

\begin{abstract}
Pituitary aspergillosis is a rare condition with only eighteen cases reported. We present the case of an immunocompetent patient found to have pituitary aspergillosis despite clinical, radiographic, and intra-operative findings consistent with pituitary macroadenoma. After surgical resection, a course of amphotericin $\mathrm{B}$, and one year of oral voriconazole our patient did not experience disease progression.
\end{abstract}

Keywords: pituitary, aspergillosis, adenoma, transsphenoidal, fungal
Volume 10 Issue 2 - 2018

\author{
Eliezer Kinberg, Caitlin Pacheco, Katelyn \\ Stepan, Hongyan Zou, Mary Fowkes, Alfred \\ lloreta
}

Mount Sinai Hospital, USA

Correspondence: Eliezer Kinberg, Resident Physician, Mount Sinai Hospital, I Gustave L. Levy Place, Annenberg 10-40, New York, NY 10029, USA, Tel (56I) 809-7857,

Email Eliezer.Kinberg@mountsinai.org

Received: December 27, 2017 | Published: April 23, 2018

\section{Introduction}

Pituitary aspergillosis is a rare condition with only eighteen cases reported. It is often initially misdiagnosed as a pituitary adenoma based on initial clinical presentation and diagnostic imaging. Fungal etiology is usually discovered intraoperatively when the lesion is visualized, but even then the classic fungal ball appearance may be absent resulting in a delayed diagnosis on histology. Once suspected, a variety of aggressive antifungal systemic therapies are typically begun post-operatively until speciation is obtained and targeted therapy can be offered. Grocott's methenamine-silver stain assists with final diagnosis by assessing for the presence of septated hyphae consistent with Aspergillus spp. Final speciation requires a fungal culture.

\section{Methods}

A chart review was performed. PubMed and Google Scholar were searched for prior cases of pituitary aspergillosis using the keywords pituitary, aspergillosis, aspergillus, and fungal. A total of 26 cases were found. Six did not involve intracranial pathology and were excluded. Two were not available in the English language and were excluded as well.

\section{Results}

After treatment with surgical excision, two weeks of intravenous Amphotericin B, and continued oral voriconazole, our patient did well and her disease has not recurred. However, there was no improvement of her pre-operative vision loss.

\section{Case presentation}

We report the case of a 67-year-old female patient who presented to her primary care physician with persistent non-specific headache. An initial MRI brain showed no overt pathology and she was treated symptomatically. Three months later, she developed progressive diplopia and blurry vision in her right eye and was found to have normal visual acuity with binocular horizontal diplopia and right orbit esotropia in primary gaze. A repeat MRI brain revealed a pituitary mass measuring $2.4 \mathrm{~cm}$, radiographically consistent with a pituitary macroadenoma. Over a two-week span, her visual symptoms rapidly progressed she presented to the emergency department for further evaluation.

Ophthalmologic exam revealed loss of light perception from the right eye and complete right abducens nerve palsy. She was initially diagnosed with pituitary apoplexy secondary to an enlarging adenoma and was scheduled for immediate surgery. Preoperative testing showed no abnormalities of pituitary endocrine function. The immediate preoperative MRI is shown in Figure 1.

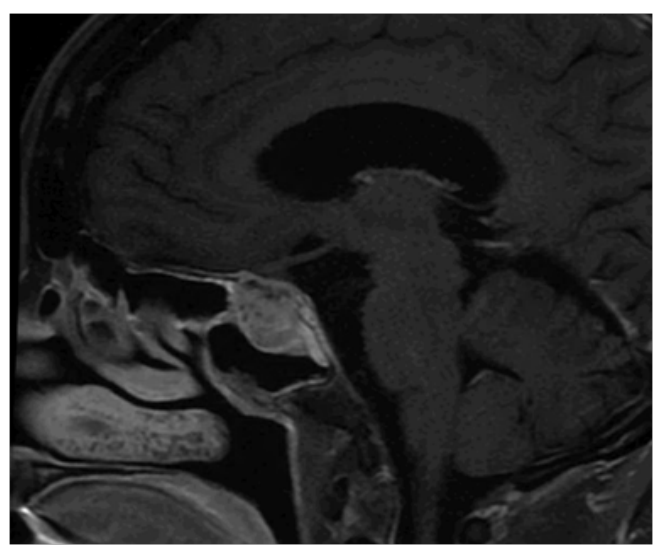

Figure I Pre-operative sagittal TI post-contrast MRI shows a $2.7 \times 2.1 \times 2.0 \mathrm{~cm}$ enhancing sellar and suprasellar mass with internal cystic components. There is upward impingement on the optic chiasm and encasement with narrowing of the right cavernous carotid.

Intraoperatively, a trans-nasal trans-sphenoidal endoscopic approach was utilized. A tan-pink mass with multiple fibrous septations was identified protruding through the floor of the sella. The gross appearance was not inconsistent with the pre-operative diagnosis of macroadenoma. The mass was de-bulked, with residual components at the right optic canal and cavernous sinus determined unresectable and left in situ. A small cerebrospinal fluid leak was noted, which was successfully repaired with a nasoseptal flap. Immediate 
postoperative MRI (Figure 2) showed satisfactory decompression of the optic chiasm, although a small rind of residual mass and persistent encasement of the right cavernous sinus and right carotid artery narrowing remained.

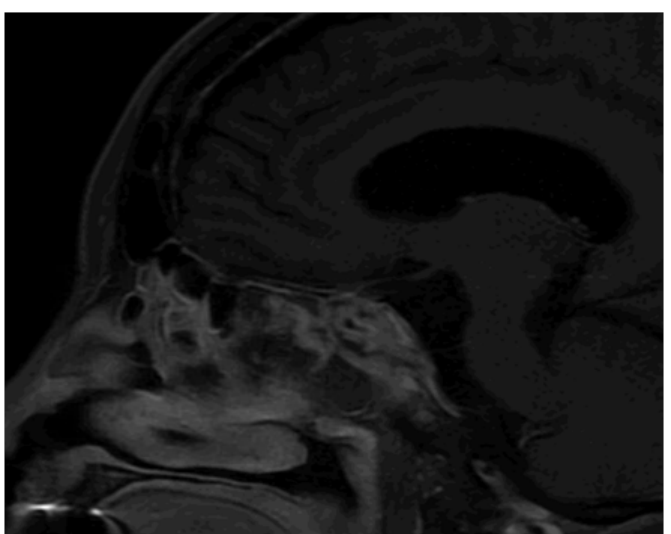

Figure 2 Sagittal TI post-contrast MRI 2 days after resection revealing heterogenous packing material in the sella, super-sella, and sphenoid sinus along with interval decompression of mass effect on the optic chiasm with a small rind of residual tumor along the inferior aspect.

Initial pathology revealed hypophysitis without features suggestive of adenoma. Further review revealed the presence of hyphae in the specimen, and a follow-up Grocott's modified methenamine-silver stain (GMS) resulted in a final diagnosis of Aspergillus spp. No cultures had been taken intraoperatively as there were no signs of infection.

The patient's standard steroid regimen was stopped on postoperative day nine after discovery of hyphae in her specimen. Two days later, she presented to the ED with weakness and instability due to secondary adrenal insufficiency and was admitted to the hospital. She was medically stabilized, and treatment was initiated with IV amphotericin B for a two-week course. After the diagnosis of Aspergillus spp. was confirmed by PCR, she was transitioned to oral voriconazole for a planned course of 6 months. There was no evidence of recurrence. Her headache resolved, however her visual loss and abducens nerve palsy did not improve largely unchanged.

Post-operative imaging at three and six months showed stable residual enhancing soft inflammatory tissue in the right cavernous sinus with extension toward the right optic canal. Additionally, there remained a focal severe stenosis of the right internal carotid artery at the distal cavernous and proximal supraclinoid segment with good reconstitution distally from the contralateral side. Given the persistent imaging findings, the patient was continued on voriconazole for a total course of one year.

\section{Discussion}

A review of the eighteen published cases reveal that pituitary fungal infection is frequently initially misdiagnosed as pituitary macroadenoma. However, an infectious pathology often becomes apparent during surgery as pus, debris, necrosis, and inflammation are found. Thirteen published cases report a pre-operative diagnosis, only two of which suspected an infectious etiology prior to surgery. ${ }^{1,2}$ Fourteen cases report intraoperative findings suspicious for infection. In the case we present, the fibrous septated mass was not suspicious for infection on gross exam. The appearance of our specimen is similar to the report by Furtado et al. which described the presence of a firm, avascular lesion. ${ }^{3}$

Notably few of the reviewed cases report endocrine abnormalities. Visual deficits were prominent for twelve patients, while only six cases detailed abnormal pituitary function. ${ }^{4-9}$ The visual deficits often present and progress quickly and rarely improve after treatment.

Confirmation of fungal etiology is often delayed due to lack of pre-operative and intra-operative suspicion for an infectious etiology. Once Aspergillus spp. is confirmed, treatment with oral voriconazole is considered appropriate therapy. Two cases ${ }^{5,10}$ did report long-term resolution without systemic therapy. ${ }^{11}$ However, intracranial fungal infection tends to have a high mortality rate with five of the eighteen reported cases resulting in death shortly after diagnosis.

There are several mechanisms classically though to account for sellar Aspergillus colonization: direct invasion from the paranasal sinuses, hematogenous dissemination from another site such as the respiratory tract, or by iatrogenic introduction during a surgical procedure. Interestingly, our patient had never had skull base or intracranial surgery and had no evidence of sinus or respiratory tract disease making these mechanisms an unlikely source of her infection. Furthermore, aspergillus classically presents in immunocompromised patients which our patient was not. The underlying source of infection in the case we present is therefore unclear and highlights the importance of considering a diagnosis of aspergillus in patients without the classic risk factors.

\section{Conclusion}

Sellar aspergillosis is a rare condition which is often initially misdiagnosed as an adenoma and can have a varied appearance intraoperatively. Early identification and treatment is imperative, and it is therefore important to consider the possibility of sellar aspergillosis even in patients without underlying risk factors. Grocott's methenamine-silver stain (GMS) positive for septated hyphae increases the likelihood of an Aspergillus diagnosis, and PCR can be used as a confirmatory test. Individually tailored treatment with oral voriconazole appears to be effective after surgical excision.

\section{Acknowledgements}

None.

\section{Conflict of interest}

The authors declare no conflict of interest.

\section{References}

1. Liu W, Chen H, Cai B, et al. Successful treatment of sellar aspergillus abscess. J Clin Neurosci. 2010;17(12):1587-1589.

2. Iplikcioglu AC, Bek S, Bikmaz K, et al. Aspergillus pituitary abscess. Acta Neurochir (Wein). 2004;146(5):521-524.

3. Furtado SV, Venkatesh PK, Ghosal N, et al. Invasive Sphenocavernous Aspergilloma Complicating an Operated Case of Acromegaly. Skull Base Rep. 2011;1(1):33-37.

4. Larrañaga J, Fandiño J, Gomez-Bueno J, et al. Aspergillosis of the sphenoid sinus simulating a pituitary tumor. Neuroradiology. 1989;31(4):362-363.

5. Boutarbouch M, Arkha Y, Ouahabi A Derraz S, et al. Sphenoid sinus aspergillosis simulating pituitary tumor in immunocompetent patient. $J$ Clin Neuroscience. 2008;16(6):840-841. 
6. Bridenstine M, Kerr JM, Lillehei KO, et al. Cushing's disease due to mixed pituitary adenoma-gangliocytoma of the posterior pituitary gland presenting with Aspergillus sp. sinus infection. Clin Neuropathol. 2013;32(5):377-383.

7. Goldhammer Y, Smith JL, Yates BM. Mycotic intrasellar abscess. American Journal of Ophthalmology. 1974;78(3):478-484.

8. Hao L, Jing C, Bowen C, et al. Aspergillus sellar abscess: case report and review of the literature. Neurol India. 2008;56(2):186-188.
9. Ouyang T, Zhang N, Wang L, et al. Primary Aspergillus Sellar Abscess Simulating Pituitary Tumor in Immunocompetent Patient. The $J$ Craniofac Surg. 2015;26(2):e86-e88.

10. Lie J, You C, Tang J, et al. Fungal pituitary abscess: Case report and review of the literature. Neurol India. 2013;61(2):210-212.

11. Petrick M, Honegger J, Daschner F, et al. Fungal granuloma of the sphenoid sinus and clivus in a patient presenting with Cranial Nerve III paresis: Case report and review of the literature. Neurosurgery. 2003;52(4):955-959. 